\title{
ABOUT THE UNIVERSAL COVERING OF THE COMPLEMENT OF A COMPLETE QUADRILATERAL ${ }^{1}$
}

\author{
WILHELM STOLL
}

In 1960, Professor Chern asked me if the universal covering of the complement of a complete quadrilateral $Q$ in the two-dimensional complex projective space $P^{2}$ were biholomorphic equivalent to a bounded domain in $\boldsymbol{C}^{2}$. The answer is negative. A recent discussion in Berkeley disclosed that this fact still seems to be unknown. Therefore, the simple proof shall be finally published. At first, a more general theorem shall be proved:

THEOREM 1. Let $M$ be a complex space. Let $\pi: \hat{M} \rightarrow M$ be an unrestricted covering space of $M$. Let $g: \hat{M} \rightarrow C^{n}$ be a holomorphic map of fiber dimension zero. Suppose that a nonconstant holomorphic map $f: C-\{0\} \rightarrow M$ exists. Then, the image $g(\hat{M})$ of $g$ is unbounded.

REMARKS TO THE TERMINology. $\pi: \hat{M} \rightarrow M$ is called a covering if $\pi$ is surjective and locally biholomorphic. The covering is called unrestricted (or regular by Ahlfors and Sario) if, for every $a \in M$, every $b \in \pi^{-1}(a)$ and every continuous map $\gamma: I \rightarrow M$ of the unit interval $I$ into $M$ with $\gamma(0)=a$, a continuous map $\tilde{\gamma}: I \rightarrow \hat{M}$ with $\tilde{\gamma}(0)=b$ and $\pi \circ \tilde{\gamma}=\gamma$ exists, i.e. if the curves of $M$ lift into $\hat{M}$. The holomorphic map $g$ is said to be of fiber dimension zero if the inverse images $g^{-1}(y)$ consist of isolated points only, or are empty.

Proof of Theorem 1. Let $\epsilon: C \rightarrow C-\{0\}$ be the exponential map: $\epsilon(z)=e^{z}$. Because $C$ is simply connected, a holomorphic map $h: C \rightarrow \hat{M}$ exists such that $\pi \circ h=f \circ \epsilon$. Suppose that $g(\hat{M})$ is bounded. Then $g \circ h$ is a bounded holomorphic vector function on C. By Liouville's theorem, $g \circ h$ is constant. Hence, a point $c \in C^{m}$ exists such that the connected set $h(\boldsymbol{C})$ is contained in $g^{-1}(c)$, whose components are points. Therefore, $h$ is constant. Hence $f \circ \epsilon=\pi \circ h$ is also constant. Because $\epsilon$ is surjective, $f$ is constant. Contradiction:

Q.E.D.

THEOREM $2 .^{2}$ Let $Q$ be the union of 4 projective lines in general position in the two-dimensional complex projective space $P^{2}$. Then the universal

Received by the editors November 22, 1968.

1 This research was partially supported by the National Science Foundation under grant NSF GP-7265.

${ }^{2}$ ADDED IN PROOF. In the meanwhile, I was informed that Peter Kiernan, Berkeley, has proved the same theorem, which will be published in these Proceedings. 
covering $\pi: \hat{M} \rightarrow M$ of $M=P^{2}-Q$ is not biholomorphically equivalent to an open bounded subset of $\mathbf{C}^{2}$.

Proof. Suppose a biholomorphic map $g: \hat{M} \rightarrow H$ onto an open bounded subset $H$ of $C^{2}$ exists. Let $L$ be a diagonal of $Q$. In an appropriate coordinate system, $M$ and $M \cap L$ are given by

$$
\begin{aligned}
M & =\left\{(z, w) \in C^{2} \mid z w(z+w-1) \neq 0\right\}, \\
f: C-\{0\} & \rightarrow L \cap M \text { biholomorphically by } f(z)=(z,-z) .
\end{aligned}
$$

Hence, $f: C-\{0\} \rightarrow M$ is not constant. Theorem 1 implies that $g(\hat{M})=H$ is not bounded. Contradiction: Q.E.D.

Of course, the proof shows that any unrestricted covering of $P^{2}-Q$ cannot be spread as a ramified covering space over a bounded subset of any $\boldsymbol{C}^{m}$. However, Chern's original conjecture becomes true if a diagonal is removed:

Proposition 3. The dicylinder is the universal covering of $A=P^{2}-(Q \cup L)$, where $L$ is a diagonal of the quadrilateral $Q$.

Proof. Observe that $E=\{z \in C \mid z \neq 0,1\}$ has the unit disk $D=\{z|| z \mid<1\}$ as universal covering. For an appropriate coordinate system is

$$
A=\left\{(z, w) \in C^{2} \mid z w(w+z-1)(w+z) \neq 0\right\} .
$$

A biholomorphic map $f: A \rightarrow E \times E$ is defined by

$$
f(z, w)=(1 /(z+w), z /(z+w)) .
$$

Therefore, $D \times D$ is the universal covering of $A$. Q.E.D.

The question of whether an open subset of $\boldsymbol{C}^{2}$ is a universal covering of $P^{2}-Q$ remains unsettled. (See Problem 25, p. 308, Proceedings of the conference on complex analysis (Minneapolis 1964), Springer-Verlag, Berlin, 1965.)

University of Notre DAME 